\title{
Impacts of ionic liquid capping on morphology and photocatalytic performance of $\mathrm{SbPO}_{4}$ crystals
}

\author{
ShunqiangChen, ${ }^{a}$ Yutong $\mathrm{Di},{ }^{\mathrm{a}}$ Taohai $\mathrm{Li}^{* a}$, Feng $\mathrm{Li}^{\mathrm{a}}$ and Wei Cao ${ }^{\mathrm{b}}$ \\ Received (in $X X X, X X X)$ Xth $X X X X X X X X X 20 X X$, Accepted Xth $X X X X X X X X X 20 X X$ \\ ${ }_{5}$ DOI: 10.1039/b000000x
}

By using [BMIM] $\left[\mathrm{PO}_{4}\right]$ as a coupling agent, a novel hydrothermal method is developed to prepare microspherical $\mathrm{SbPO}_{4}$ with different morphologies and photocatalytic abilities. An evolution from flower- to sphere-shaped products was observed. Regular $\mathrm{SbPO}_{4}$ microspheres with diameters of 10-35 $\mu \mathrm{m}$ were found to exhibit excellent photocatalytic properties to degrade the rhodamine B (45 min, $99 \%$ ) and methylene blue (60 min, $99 \%)$ under UV light irradiation. Impacts of the ionic liquid type, capping abilities and reaction 10 conditions on final products were revealed. It is found that by tuning reaction time and temperature, the [BMIM][PO4] can gradually react with $\mathrm{SbCl}_{3}$, forming the $\mathrm{SbPO}_{4}$ products with controllable morphology of and tailored bandgap. Distinguished photocatalytic abilities are attributed to large surface area and low bandgap energy of the semiconductor.

\section{Introduction}

Photocatalytic degradation of toxic organic pollutants has drawn 15 great interests due to its environmentally friendly merits. ${ }^{1-3}$ To date, photocatalysts are emphasized on semiconductor materials thanks to their promising photodegrade ability of organic contaminants $^{4}$ and solar energy conversion. ${ }^{5}$ These semiconductor materials can be endowed with narrow bandgap,

20 large surface area, specific morphology and structure through proper synthetic routes, such as the hydrothermal method.,7 Conventionally, photocatalytic materials are limited within semiconductors containing $\mathrm{TiO}_{2},{ }^{8} \mathrm{In}_{2} \mathrm{O}_{3},{ }^{9}$ and $\mathrm{ZrO}_{2},{ }^{10}$ etc. Recent researches showed that other chemical composites containing ${ }_{25} \mathrm{Bi}_{2} \mathrm{O}_{3},{ }^{11} \mathrm{SnO}_{2},{ }^{12}$ and $\mathrm{Sb}_{2} \mathrm{~S}_{3},{ }^{13}$ are also functional as active sites for photocatalysis. $^{14}$ Among them, the antimony-based materials have been extensively investigated because of their excellent optical properties ${ }^{15}$ and potential applications in sunlight harvesting. ${ }^{16}$ Numerous efforts were elaborated in studies of the ${ }_{30} \mathrm{Sb}$-based photocatalysts, such as $\mathrm{Sb}_{2} \mathrm{~S}_{3},{ }^{16} \mathrm{Sb}_{2} \mathrm{WO}_{6},{ }^{17} \mathrm{Sb}_{2} \mathrm{O}_{3},{ }^{18}$ and $\mathrm{M}_{2} \mathrm{Sb}_{2} \mathrm{O}_{7}(\mathrm{M}=\mathrm{Ca}, \mathrm{Sr}),{ }^{14}$ etc. The $\mathrm{SbPO}_{4}$ microsphere with 4.42 $\mathrm{eV}$ of band gap energy is a new promising photocatalyst under UV light irradiation. ${ }^{9}$ Despite of a few reports on its preparation through hydrothermal reaction ${ }^{19}$ and solvothermal decomposition 35 method, ${ }^{20}$ improvements of material's synthesis routes and photocatalytic functionality are scarcely reported.

Besides electronic structures, photocatalytic ability of the semiconductors also depends on materials size, morphology, surface area and structure. In this regard, the hydrothermal 40 synthesis is advantageous in controlling these extrinsic properties $^{21}$, benefitting the final photocatalytic performances of the $\mathrm{SbPO}_{4}$. Proper media and conditions are essential during the wet synthesis. ${ }^{6}$ It is noticed that the ionic liquids (ILs) are widely employed as the reagents in other compound preparations due to 45 their remarkable thermal and chemical stability, dissolving ability, high ionic conductivity and low potentiallytoxicity. ${ }^{22,23}$ As a capping agent and phosphorus source, the 1-butyl-3methylimidazolium phosphate ([BMIM]P) ionic liquid plays an important role in the facile and efficient synthesis of the $\mathrm{SbPO}_{4}{ }^{24}$

${ }_{50}$ Inspired by these progresses, the hydrothermal synthesis of $\mathrm{SbPO}_{4}$ is naturally extended to use the $[\mathrm{BMIM}]\left[\mathrm{PO}_{4}\right]$ as a new phosphors source, and vary reaction parameters to tailor product properties.

In this paper, we report on a facile hydrothermal method to ${ }_{55}$ prepare $\mathrm{SbPO}_{4}$ with the [BMIM][PO4] as a coupling agent, and study its impact on the morphology and catalytic ability of the final products. The morphological evolution of the products is studied. The photocatalytic ability was evaluated by degrading rhodamine $\mathrm{B}(\mathrm{RhB})$ and methylene blue $(\mathrm{MB})$ under UV light

${ }_{60}$ irradiation. It is found that the homogeneous $\mathrm{SbPO}_{4}$ microspheres can eliminate these dyes within an hour timespan.

\section{Experimental Section}

\section{Materials}

Reagents of the 1-butyl-3-methylimidazolium phosphate 65 ([BMIM] $\left.\left[\mathrm{PO}_{4}\right]\right), \mathrm{SbCl}_{3}, \mathrm{Na}_{2} \mathrm{HPO}_{4} \cdot 12 \mathrm{H}_{2} \mathrm{O}, \mathrm{RhB}, \mathrm{MB}$ were of analytical grade and used without further purification. All the chemicals in our experiments were purchased from Shanghai Chemical Reagents Company (China).

\section{Catalysts preparation.}

70 In a typical process, $2 \mathrm{mmol}$ of [BMIM] $\left[\mathrm{PO}_{4}\right]$ was added in 15 $\mathrm{mL}$ water and continuously stirred for $10 \mathrm{~min}$ at room temperature until a homogeneous solution was obtained. Then, 2 mmol of $\mathrm{SbCl}_{3}$ was quickly added into the beaker. The mixture was magnetically stirred for 30 minutes. It was transferred into a

75 Teflon-lined autoclave, kept at $160^{\circ} \mathrm{C}$ for $24 \mathrm{~h}$ and cooled down to room temperature naturally. The resulted white-colored precipitate was separated by centrifugation, washed with deionized water and absolute ethanol 3 times, respectively. It was transferred to an oven and dried at $70^{\circ} \mathrm{C}$ for $12 \mathrm{~h}$. The collected 80 product was named as S-P-2.

To study morphology impacts on photocatalytic reactivity, other $\mathrm{SbPO}_{4}$ samples were also fabricated by varying reaction temperature (S-P-1, S-P-3), Sb/P mole ratios (S-M-1, S-M-2) and reaction time (S-T-1, S-T-2). The detailed reaction parameters 85 were tabulated in Table 1 . The $\mathrm{SbPO}_{4}$ with other organic phosphate salt (1-ethyl-3-methylimidazolium methyl phosphate) and the $\mathrm{SbPO}_{4}$ without ILs were also prepared in the same procedure. The obtained sample was designated as S-O-1, S-O-2, S-O-3 and S-P-4. 
Table 1. Experimental parameters for the synthesis of $\mathrm{SbPO}_{4}$

\begin{tabular}{|c|c|c|c|c|}
\hline Sample & Reactants & & Temperature & Time \\
\hline S-P-1 & $2 \mathrm{mmol}$ of $[\mathrm{BMIM}]\left[\mathrm{PO}_{4}\right]$, & $2 \mathrm{mmol}$ of $\mathrm{SbCl}_{3}$ & 140 & 24 \\
\hline S-P-2 & $2 \mathrm{mmol}$ of $[\mathrm{BMIM}]\left[\mathrm{PO}_{4}\right]$, & $2 \mathrm{mmol}$ of $\mathrm{SbCl}_{3}$ & 160 & 24 \\
\hline S-P-3 & $2 \mathrm{mmol}$ of $[\mathrm{BMIM}]\left[\mathrm{PO}_{4}\right]$, & $2 \mathrm{mmol}$ of $\mathrm{SbCl}_{3}$ & 180 & 24 \\
\hline S-P-4 & $2 \mathrm{mmol}$ of $\mathrm{Na}_{2} \mathrm{HPO}_{4} \cdot 12 \mathrm{H}_{2} \mathrm{C}$ &, $2 \mathrm{mmol}$ of $\mathrm{SbCl}_{3}$ & 160 & 24 \\
\hline S-M-1 & $1 \mathrm{mmol}$ of $[\mathrm{BMIM}]\left[\mathrm{PO}_{4}\right]$, & $2 \mathrm{mmol}$ of $\mathrm{SbCl}_{3}$ & 160 & 24 \\
\hline S-M-2 & $3 \mathrm{mmol}$ of $[\mathrm{BMIM}]\left[\mathrm{PO}_{4}\right]$, & $2 \mathrm{mmol}$ of $\mathrm{SbCl}_{3}$ & 160 & 24 \\
\hline S-T-1 & $2 \mathrm{mmol}$ of $[\mathrm{BMIM}]\left[\mathrm{PO}_{4}\right]$, & $2 \mathrm{mmol}$ of $\mathrm{SbCl}_{3}$ & 160 & 12 \\
\hline S-T-2 & $2 \mathrm{mmol}$ of $[\mathrm{BMIM}]\left[\mathrm{PO}_{4}\right]$, & $2 \mathrm{mmol}_{\text {of }} \mathrm{SbCl}_{3}$ & 160 & 48 \\
\hline S-O-1 & $2 \mathrm{mmol}$ of [EMIM]DMP, & $2 \mathrm{mmol}$ of $\mathrm{SbCl}_{3}$ & 160 & 12 \\
\hline S-O-2 & $2 \mathrm{mmol}$ of [EMIM]DMP, & $2 \mathrm{mmol}$ of $\mathrm{SbCl}_{3}$ & 160 & 24 \\
\hline S-O-3 & $2 \mathrm{mmol}$ of [EMIM]DMP, & $2 \mathrm{mmol}$ of $\mathrm{SbCl}_{3}$ & 160 & 48 \\
\hline
\end{tabular}

\section{Characterization}

The crystal structure of the $\mathrm{SbPO}_{4}$ was studied through the X-ray 5 diffraction (XRD) patterns on an X-ray diffractometer (XRD, $\mathrm{D} / \mathrm{max}-\mathrm{RB}$, Rigaku, Japan) with the $\mathrm{Cu}-\mathrm{K} \alpha$ radiation $(\lambda=0.15418$ $\mathrm{nm})$ as the incident source. The surface morphology was recorded through the scanning electron microscopy (SEM, JSM-6700F, JEOL, Japan), while grain sizes were obtained via the 10 transmission electron microscopy (TEM, JEM-2100F, JEOL, Japan). The Brunauer-Emmett-Teller (BET) surface area was examined on an ASAP 2010 Brunauer-Emmett-Teller (BET) analyzer. Element compositions were determined through the energy dispersive X-ray spectroscopy (EDX, EX-250, Horiba, 15 Japan). Chemical states of elements were studied via X-ray photoelectron spectroscopy carried out through the XPS Escalab 250, Thermo Scientific, USA. Bandgaps the products were deduced from UV-vis spectra which were performed on a UVvis spectrophotometer (Lambda 950, Perkin-Elmer, USA). The 20 photoluminescence (PL) spectra were measured on a HitachiF7000 spectrophotometer with the excitation wavelength of 220 $\mathrm{nm}$.

\section{Measurements of photocatalytic activity}

The photocatalytic abilities were determined by the RhB and MB 25 degradation. A $500 \mathrm{~W}$ UV-C lamp was used as the light source and placed $8 \mathrm{~cm}$ from the reactor. With vigorous stirring, $50 \mathrm{~mL}$ of $\mathrm{RhB}$ (or $\mathrm{MB}$ ) (10 mg/L) aqueous solution was mixed with 0.05 gas-prepared photocatalysts. Prior to photoreaction, the solution was stirred in dark for about $30 \mathrm{~min}$ to reach the $\mathrm{RhB}$ (or $30 \mathrm{MB}$ ) adsorption-desorption equilibrium. At regular intervals, 3 $\mathrm{mL}$ samples were taken and filtered to remove the particles. The concentration of $\mathrm{RhB}$ (or $\mathrm{MB}$ ) was determined by monitoring peaks of RhB at $552 \mathrm{~nm}$ (or MB at $664 \mathrm{~nm}$ ) measured by a UVVis spectrophotometer (Lambda 950, Perkin-Elmer, USA).

\section{${ }_{35}$ Results and discussion}

\section{X-ray diffraction.}

Figure 1 shows the XRD patterns of $\mathrm{SbPO}_{4}$ (S-P-1, S-P-2, S-P-3) prepared with the assistance of the $[\mathrm{BMIM}]\left[\mathrm{PO}_{4}\right] \mathrm{IL}$, and the one without IL (S-P-4). Sharp characteristic peaks locate at the $2 \theta$ 40 angles of $21.8^{\circ}, 22.9^{\circ}, 24.6^{\circ}, 26.3^{\circ}, 26.7^{\circ}, 29.8^{\circ}, 35.2^{\circ}, 36.3^{\circ}$, $37.8^{\circ}, 43.8^{\circ}$ and $54.3^{\circ}$. They are well indexed to the monoclinic $\mathrm{SbPO}_{4}$ (JCPDS Card NO. 35-0829, space group P21/m, unit-cell parameters $a=5.106$ (2) $\AA, b=6.772$ (1) $\AA, c=5.092$ (2) $\AA$ ), denoting that these products are monoclinic structure with high 45 degree of crystallinity.

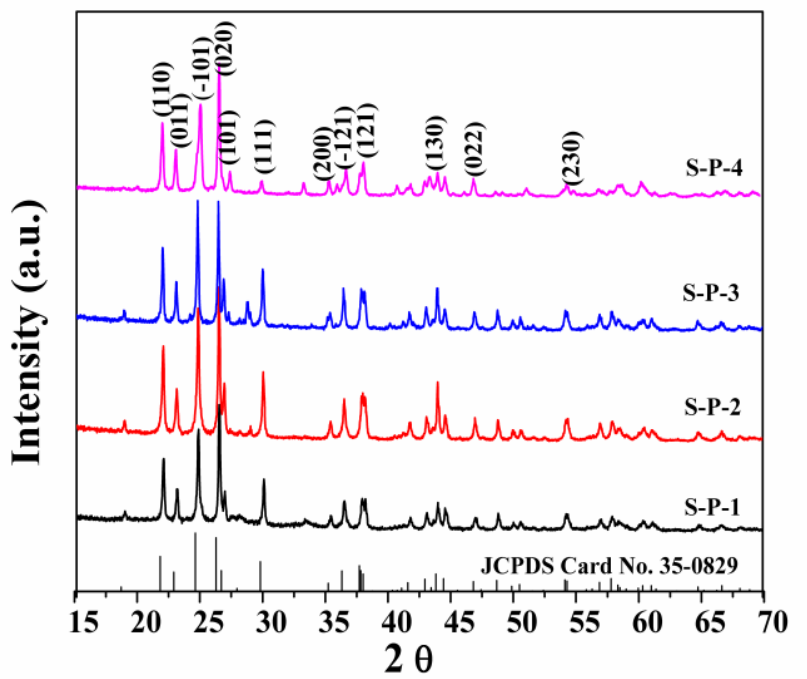

Figure 1. XRD patterns of $\mathrm{SbPO}_{4}$ samples prepared at different hydrothermal parameters.

The XRD patterns of $\mathrm{SbPO}_{4}$ prepared at different $\mathrm{Sb} / \mathrm{P}$ mole ratio 50 and hydrothermal time are shown in Figure S1, S2 at the Electronic Supplementary Information (ESI). Some additional diffraction peaks with $2 \theta$ at $33.1^{\circ}$ and $29.2^{\circ}$ are observed, which are indexed to the $\mathrm{Sb}\left(\mathrm{PO}_{4}\right)_{3}(\mathrm{JCPDS}$ Card NO. 51-0234)and $\mathrm{SbOPO}_{4}($ JCPDS Card NO. 40-0037).

55

\section{SEM and HRTEM analysis.}

Figure 2 shows the SEM images of the as-prepared samples. From the figure, the diameter of the microspheres was found around 10-35 um. At the beginning of forming stage (S-P-1, ${ }_{60}$ Figure 2a) the sample was flower-like $\mathrm{SbPO}_{4}$ microspheres with diameters of $32-38 \mu \mathrm{m}$. The well-dispersed product was composed of primary nanorods and nanoplates as observed on the rough surface. With the increase of the reaction temperature, the diameters of the flower-like microspheres decrease to 28-33 $\mu \mathrm{m}$.

${ }_{65}$ As shown in Fig. 2c, the surfaces of these flower-like microspheres are smoother than that of S-P-1. When After adjusting synthesis temperature to $180{ }^{\circ} \mathrm{C}(\mathrm{S}-\mathrm{P}-3$, Figure $2 \mathrm{c})$ the microflowers evolved to sphere-like $\mathrm{SbPO}_{4}$ with partially broken and shriveled debris. It can be seen clearly from Figure $2 \mathrm{~d}$ that 70 compared to the flower-like microspheres (Figure $2 a, b, c$ ), increasing the temperature of the reaction would result in the decrease of product's diameter. Furthermore, the morphologies and surface roughness have obvious changes compared with microflowers. The initial flower-like structure formed by 75 nanorods and nanoplates exhibits a high porosity, and eventually turned into a compacted sphere-like structure with a smooth surface. Compared to the ionic-liquid-assisted $\mathrm{SbPO}_{4}$, the nonionic-liquid-assisted $\mathrm{SbPO}_{4}$ shown in Figure 2d are rather consisted of nanorods and nanoplates. 

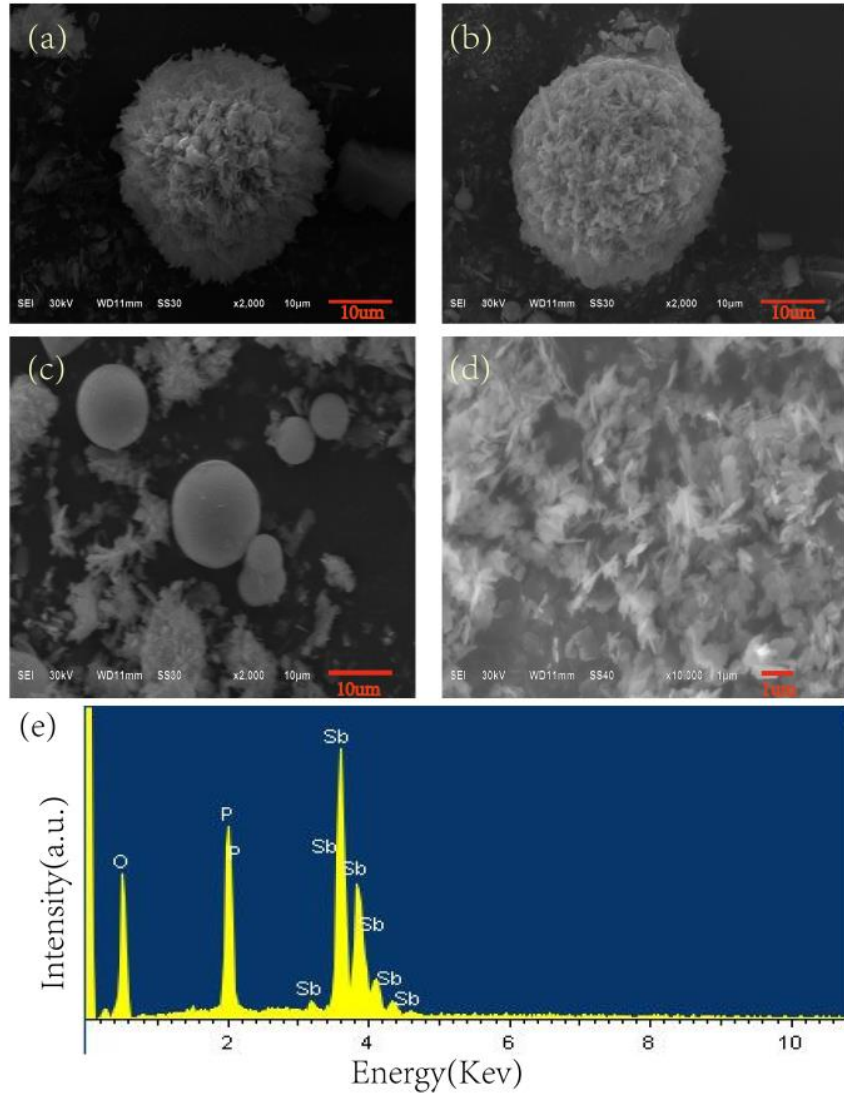

Figure 2. SEM images of the $\mathrm{SbPO}_{4}$ synthesized at different temperatures: (a) S-P-1, (b) S-P-2, (c) S-P-3, (d) S-P-4,

(e) EDX spectrum of S-P-2.

${ }_{5}$ Indeed, similar morphological transformations were observed in other synthetic crystal systems. ${ }^{19,25}$ Moreover, the change of the $\mathrm{Sb} / \mathrm{P}$ mole ratio can also result in similar evolution (see Fig. S3 in ESI). The EDX analysis of S-P-2 (Figure 2e) confirms the elements of $\mathrm{Sb}, \mathrm{P}$ and $\mathrm{O}$ at a ratio of $1: 1: 4$, very near to the 10 stoichiometric value.

Figure 3 shows high-magnification SEM, the TEM, high resolution TEM (HRTEM) images and selected area electron diffraction (SAED) patterns of S-P-2. The TEM images with more detailed information (Figure $3 \mathrm{a}, \mathrm{b}$ ) reveal that

15 the S-P-2 was composed of nanorods and nanoplates, in consistent with the SEM results. As shown in Figure 3c, the HRTEM image of the microsphere clearly identify the lattice spacing of $\mathrm{SbPO}_{4}$, which matches well with the XRD analyses. The $0.17 \mathrm{~nm}$ d-spacing is corresponding to the (111) crystal plane 20 of $\mathrm{SbPO}_{4}$ microspheres. The SAED pattern (Figure 3d) of S-P-2 microspheres clearly confirms that the microsphere is only consisted of the monoclinic $\mathrm{SbPO}_{4}$.
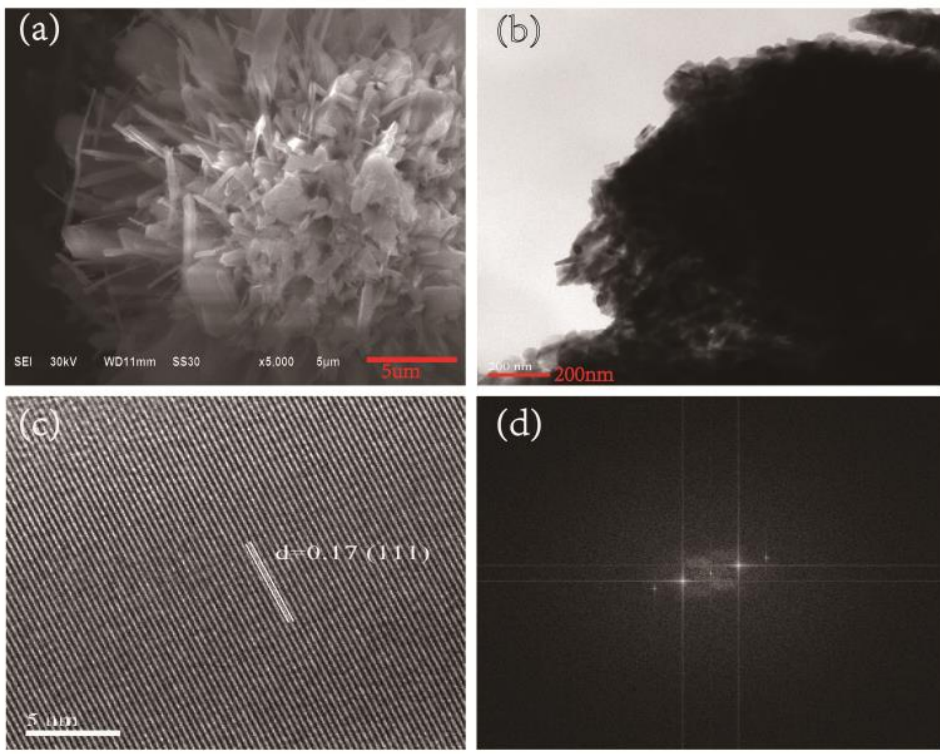

Figure 3. (a) high-magnification SEM images of S-P-2 (b) 25 TEM images of S-P-2, (c) HRTEM image of S-P-2, (d) SAED pattern of S-P-2.

Growth mechanism of $\mathrm{SbPO}_{4}$ microspheres.

We first exam the influences of preparation time on the morphology of the final product. A series of preparations 30 was performed by varying the hydrothermal time and keeping other conditions unchanged. First, the XRD characterization is used to study the phase evolution process and depicted in Fig. S11. The product is amorphous at a short reaction time of $4 \mathrm{~h}$. It turns to pure ${ }_{35} \mathrm{Sb}_{8} \mathrm{O}_{11} \mathrm{Cl}_{2}$ (JCPDS no.21-0052) when the reaction time reaches $8 \mathrm{~h}$. Moreover, the intensity of the characteristic peak $\left(2 \Theta=27.94^{\circ}\right)$ of the $\mathrm{Sb}_{8} \mathrm{O}_{11} \mathrm{Cl}_{2}$ phase gradually decrease while the one from $\mathrm{SbPO}_{4}$ increase with the reaction time. Pure $\mathrm{SbPO}_{4}$ was generated after $12 \mathrm{~h}$ of 40 hydrothermal treatment. This shows that the original product $\mathrm{Sb}_{8} \mathrm{O}_{11} \mathrm{Cl}_{2}$ has gradually transformed into $\mathrm{SbPO}_{4}$ during the IL-assisted preparation.

Morphological evolution was also studied. The SEM images of the products are shown in Figure 4, where differences in 45 morphologies bear the $\mathrm{SbPO}_{4}$ formation stages. Generally, the microspheres assembly process has been observed for the formation of the $\mathrm{SbPO}_{4}$ microspheres. ${ }^{25,}{ }^{26}$ The $2 \mathrm{D}$ nanoplates were formed in the early stages of synthesis, and stick to each other (Figure 4a). Later, the 2D nanorods and nanoplates will 50 grow to $3 \mathrm{D}$ microspheres with obviously concaves at the spherical centres of the samples (Figure 4b). The surface roughness of the as-prepared samples decreases with reaction time (12 h, 24 h, Figure 4c, d). Based on the above evidences, a probable formation mechanism of the $\mathrm{SbPO}_{4}$ microspheres was 55 concluded in the Scheme 1.

In order to investigate the influence of ILs on the controllable fabrication and morphology of the obtained $\mathrm{SbPO}_{4}$, another organic phosphate salt ([EMIM]DMP) was used as the reagent instead of the [BMIM] $\left[\mathrm{PO}_{4}\right]$. Figure S5 explicitly shows that as-

60 synthesized S-O-1, S-O-2 and S-O-3 with different hydrothermal reaction time are microrods with widths of $4.55-6.13 \mu \mathrm{m}$ and length of $15.29-23.04 \mu \mathrm{m}$. Morphologies are influenced by the species of ILs. 


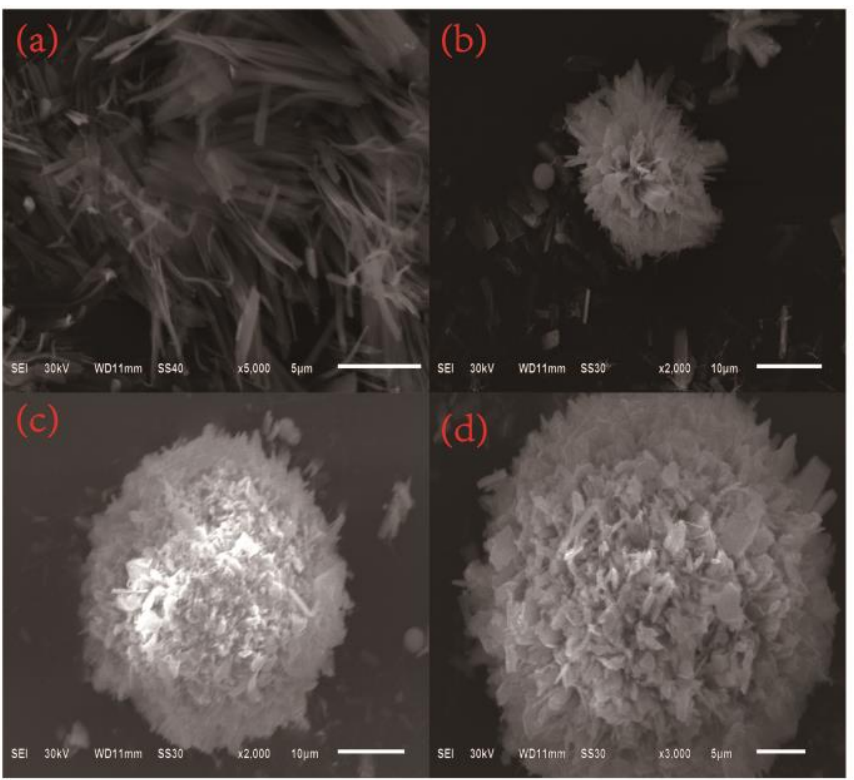

Figure 4. SEM images of the $\mathrm{SbPO}_{4}$ fabricated at different reaction time: (a) 4, (b) 8, (c)12, (d)24.

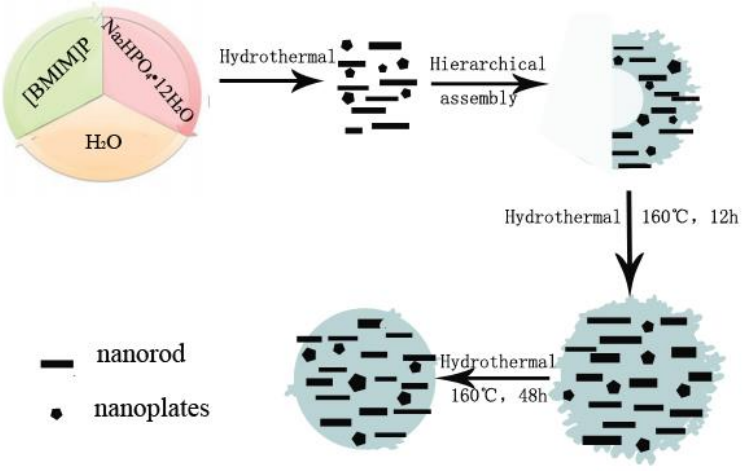

Scheme 1. The schematic illustration of the formation of $\mathrm{SbPO}_{4}$ solid microspheres.

\section{XPS analysis}

The elemental compositions and chemical states of S-P-2 were further measured by the XPS and the detailed results are 10 displayed in Figure 5. The spectral energies were calibrated by using the adventitious $\mathrm{C} 1 \mathrm{~s}$ peak at $284.8 \mathrm{eV}$. The XPS of the $\mathrm{Sb}$ $3 d_{3 / 2}$ is depicted in Figure 5a, P $2 p$ in Figure $5 b$, and the $O 1 s$ in Figure 5c. A binding energy of $540.40 \mathrm{eV}$ is assigned to the $3 \mathrm{~d}_{3 / 2}$ from the $\mathrm{Sb}^{3+}$ ions. ${ }^{27}$ Peaking at $133.76 \mathrm{eV}$, the phosphorus $2 \mathrm{p}$ 15 spectrum denotes the element is in the form of $\mathrm{P}^{5+3}$. Figure $5 \mathrm{c}$ demonstrates that the single peak of $\mathrm{O} 1 \mathrm{~s}$ is centered at 531.17 eV. ${ }^{28}$ Chemical states of the elements confirm the $\mathrm{SbPO}_{4}$ is the final product.
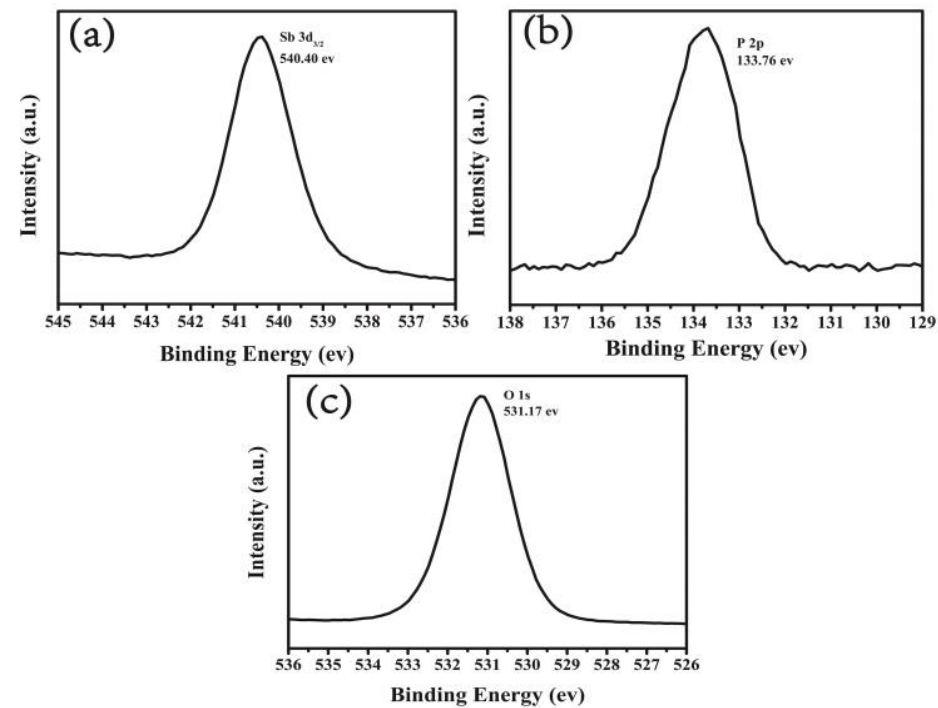

Figure 5. XPS spectra of S-P-2: (a) Sb 3d3/2 peak, (b) P 2p peak,

\section{Optical properties}

(c) $\mathrm{O} 1 \mathrm{~s}$ peak

The bandgaps of the products were determined from UV-Vis diffuse reflection spectra, as shown in Fig.6 for S-P-1, S-P-2, S25 P-3, S-P-4 and ILs. The curves exhibit a clear absorption edge of S-P-4 at about $283 \mathrm{~nm}$, in consistence with the previous results. ${ }^{19}$ However, the absorbance of S-P-1, S-P-2 and S-P-3 (Figure 6a) is obviously enhanced at the range of $300-600 \mathrm{~nm}$ but not at 650 $700 \mathrm{~nm}$ originated from the IL absorption (Figure S10). Thus, the 30 substantial red shift in the bandgap transition of the ionic-liquidassisted $\mathrm{SbPO}_{4}$ is attributed to the changes in morphology of the $\mathrm{SbPO}_{4}$ samples. Compared with S-P-3 prepared at $180^{\circ} \mathrm{C}, \mathrm{S}-\mathrm{P}-1$ and S-P-2 also show red shifts in the bandgap transitions. This is most probably attributed to the relatively defective surface as 35 shown in Fig.2. Such redshifts may also be beneficial to enable the utilization of UV-light for the photocatalytic degradation of the dyes. ${ }^{29}$

The bandgap energy (Eg) of the sample follows the equation: $\alpha=A\left((h v-E g)^{n / 2}\right) /(h v)$

40 where $\alpha, v, \mathrm{~A}$, and $\mathrm{Eg}$ are absorption coefficient, light frequency, a constant and band gap energy $(\mathrm{eV})$, respectively. Among them, the value of $n$ depends on whether the transition is direct $(n=1)$ or indirect $(n=4) .{ }^{30}$ The bandgap energies obtained from plots $(\text { absorbance) })^{2}$ versus energy (hv) ${ }^{2}$ (Figure 6b) are about 3.19, 45 2.80, 4.12 and $4.71 \mathrm{eV}$ for S-P-1, S-P-2, S-P-3 and S-P-4, respectively. A relatively low bandgap of $2.80 \mathrm{eV}$ was found for the S-P-2. It is mention worth that the adsorption band of $[\mathrm{BMIM}]\left[\mathrm{PO}_{4}\right]$ ion pair is around $550 \mathrm{~nm}(\text { Figure } \mathrm{S} 10)^{31}$, out of the regions of samples' bandgap distributions.
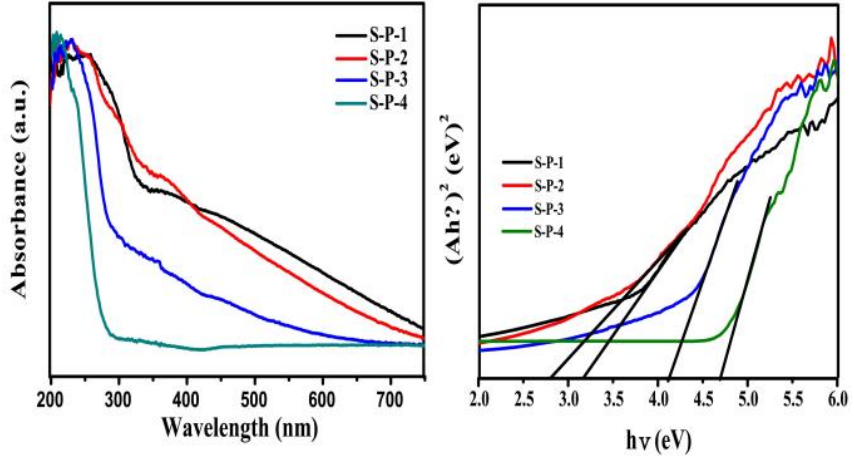

Figure 6. (a)UV-Vis DRS spectra of S-P-1, S-P-2, S-P-3 and S-P4, (b) Band gap of S-P-1, S-P-2, S-P-3 and S-P-4 
PL measurements were applied to determine the recombination behavior of electron-hole combinations after photoexcitation of the semiconductors at excitation at $220 \mathrm{~nm}^{33}$. The spectra are shown in Fig 7. Higher recombination of carriers yields lower 5 separation efficiency of carriers. All of samples show similar peak at about 540nm. It is evident that the S-P-1, S-P-2, S-P-3 obviously emit less PL intensity than the S-P-4. This implies smaller recombination rates between the photocreated electrons and holes. Among these $\mathrm{SbPO}_{4}$, the S-P-2 catalyst is the least 10 luminescent. This is attributed to the possible excitonic behaviors of the moderately defective structures of semiconductors ${ }^{[34]}$.

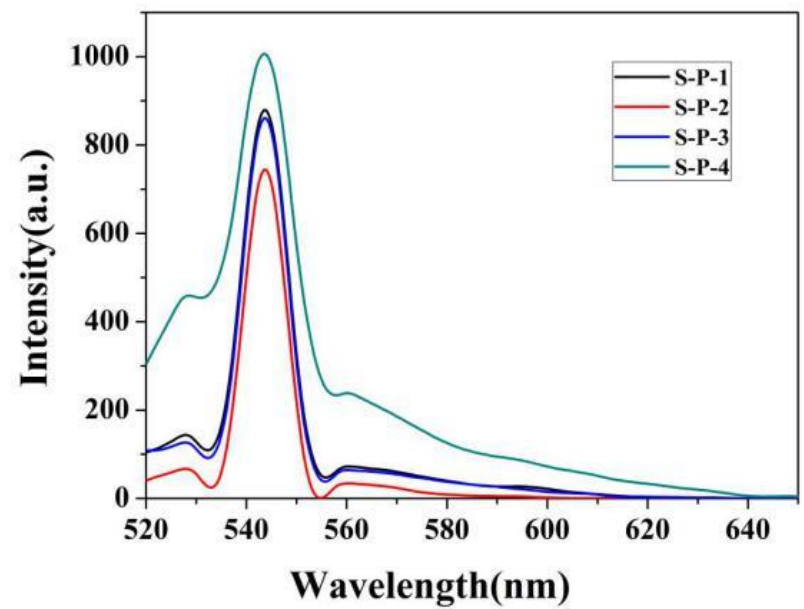

Figure 7.The PL spectra for S-P-1, S-P-2, S-P-3 and S-P-4 samples, respectively.

15

FTIR analysis

Figure 8 shows the FTIR spectra of S-P-1, S-P-2, S-P-3, S-P-4 and ILs. According to the literature, ${ }^{32}$ the two vibrations centered at $1023 \mathrm{~cm}^{-1}$ and $953 \mathrm{~cm}^{-1}$ are due to the $v_{1}$ symmetric vibrations, 20 while the vibration around $1150 \mathrm{~cm}^{-1}$ can be associated with the corresponding $v_{3}$ asymmetric stretching vibration of $\mathrm{P}-\mathrm{O}$ bond. The vibration at $610 \mathrm{~cm}^{-1}$ corresponds to the bending vibration of $\mathrm{O}-\mathrm{P}-\mathrm{O}$ linkages. However, the vibration of the ILs evidently gives a different band shape (cyan line in the figure). 25 This provides strong evidence for the absent of ILs in the asprepared samples.

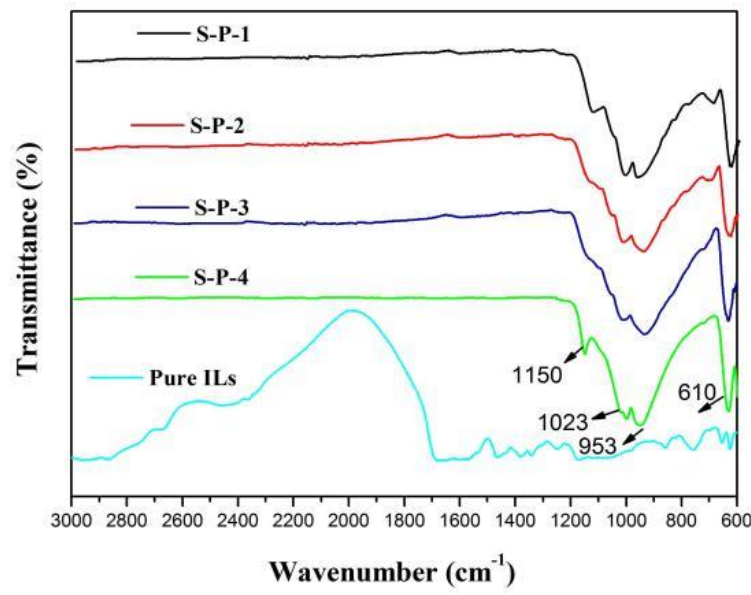

Figure 8.FTIR spectra of S-P-1, S-P-2, S-P-3, S-P-4 and ILs. BET analysis

30 The nitrogen adsorption and desorption measurements for S-P-1,
S-P-2, S-P-3 and S-P-4 are shown in Figure 9 and Table 2. From the results, the isotherm of S-P-1 and S-P-2 can be assigned to type IV with an indistinct hysteresis loop $^{35}$, which was attributed the mesoporous material. The isotherm of S-P-3 and S-P-4 35 presents II type isotherms, which demonstrates the absence of mesoporous material in the prepared samples ${ }^{35}$. The specific surface areas of S-P-1, S-P-2, S-P-3 and S-P-4 are 5.14, 6.98, 15.35 and $13.07 \mathrm{~m}^{2} / \mathrm{g}$, respectively. A slight increase in the specific surface area is observed when increasing the reaction 40 temperature. This phenomenon maybe caused by thermal defragmentation of large particles. However, compared to the specific surface area of samples, the influence of specific surface area is negligible for the overall photocatalytic activity.
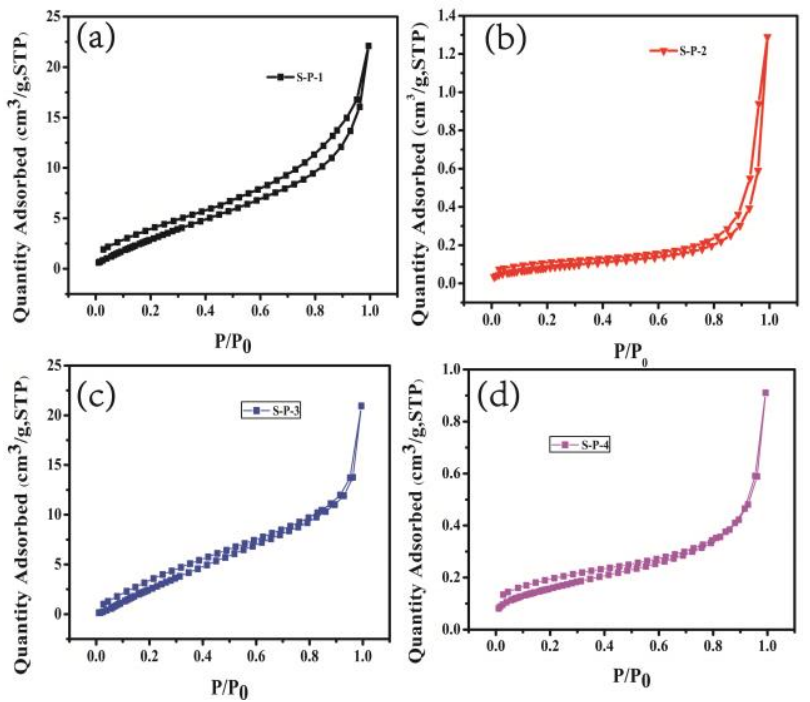

45 Figure 9. Adsorption-desorption isotherms of $\mathrm{SbPO}_{4}$ prepared at different conditions.

Table 2. Structural parameters obtained from $\mathrm{N}_{2}$ adsorption isotherms analysis.

\begin{tabular}{cccc}
\hline Sample & S $_{\text {BET }}\left(\mathrm{m}^{2} \mathrm{~g}^{-1}\right)$ & Pore volume $\left(\mathrm{cm}^{3} \mathrm{~g}^{-1}\right)$ & $\begin{array}{c}\text { Average pore size } \\
(\mathrm{nm})\end{array}$ \\
\hline S-P-1 & 5.14 & 0.032292 & 8.83273 \\
S-P-2 & 6.98 & 0.044233 & 25.6388 \\
S-P-3 & 15.35 & 0.029994 & 5.11134 \\
S-P-4 & 13.07 & 0.029351 & 9.65675 \\
\hline 50 & & &
\end{tabular}

50

\section{Photocatalytic activity}

Figure $10 \mathrm{a}$ and $\mathrm{b}$ show the time-course photocatalytic decolorations of $\mathrm{RhB}$ and $\mathrm{MB}$ over $\mathrm{SbPO}_{4}$ prepared at different reaction temperature under UV light irradiation, along with the

55 blank tests. Monitoring spectra of the photodegradation reaction of RhB and MB over S-P-2 are shown in the insets. From the figures, $\mathrm{RhB}$ and $\mathrm{MB}$ appear to be stable under UV light irradiation without the presence of $\mathrm{SbPO}_{4}$. The degradation or adsorption of $\mathrm{RhB}$ and $\mathrm{MB}$ are negligible (about 3-7\% and 1-

${ }_{60} 11 \%$ ) on the $\mathrm{SbPO}_{4}$ without UV irradiation. Obviously, introductions of $\mathrm{SbPO}_{4}$ can substantially improve the degradation rates of the dyes. The decoloration rate of $\mathrm{RhB}$ is about $86 \%$, $99 \%, 97 \%$ and $73 \%$ over S-P-1, S-P-2, S-P-3 and S-P-4 after UV light irradiation for $45 \mathrm{~min}$. Similarly, $79 \%, 99 \%, 91 \%$ and ${ }_{65} 60 \% \mathrm{MB}$ was degraded over the samples after UV light irradiation for $60 \mathrm{~min}$, respectively. All the removal efficiencies of the ionic-liquid-assisted $\mathrm{SbPO}_{4}$ are better than these of $73 \%$ and $60 \%$ from the no-ionic-liquid-assisted $\mathrm{SbPO}_{4}$. 

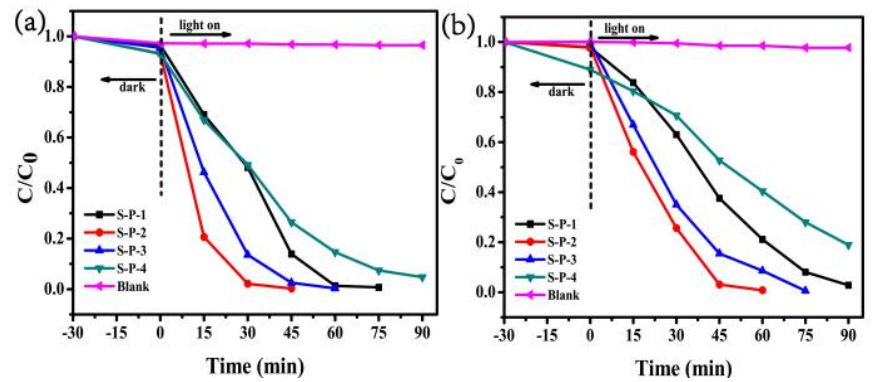

Figure 10. Photocatalytic degradation of RhB (a) and MB (b) by $\mathrm{SbPO}_{4}$ photocatalysts.

The kinetics of photocatalytic performance was also studied. The pseudo-first-order kinetic model reads

$$
\ln \left(C_{0} / C_{t}\right)=F(t)=k t
$$

where $\mathrm{k}$ is the apparent reaction rate constant, $\mathrm{C}_{0}$ and $\mathrm{C}_{\mathrm{t}}$ are the 10 initial concentration and the concentration at the reaction time of t. Natural logarithms of concentration ratios from the catalytic measurements were plotted as functions of reaction time in Figure $11 \mathrm{a}$ and $\mathrm{b}$ for the $\mathrm{RhB}$ and $\mathrm{MB}$, respectively. The logarithmic data were fitted through the above formula, and the 15 fitted lines were drawn in the same figures. The reaction rate constants of RhB over S-P-1, S-P-2, S-P-3, S-P-4 and without photocatalyst were calculated to $0.07161,0.13304,0.09496$, 0.03464 and $0.00003 \mathrm{~min}^{-1}$. These values were given in Figure $10 \mathrm{a}$. With the increase of synthesis temperature, the rate constant 20 of $\mathrm{SbPO}_{4}$ samples first increased and then decreased. As shown in Figure $11 \mathrm{~b}$, the same results can also be observed by the degradation of MB. Thus, the ionic-liquid-assisted $\mathrm{SbPO}_{4}$ microspheres exhibited higher photodegradation rate in the photocatalytic performance of RhB and MB. Especially, the S-P${ }_{25} 2$ possessed the largest rate constant during the whole period of 90 minutes of the UV irradiation. Such a value is 3.9 times of that of no-ionic-liquid-assisted $\mathrm{SbPO}_{4}$ for the degradation of $\mathrm{RhB}$. The top degradation efficiency of S-P-2 over both RhB and MB was attributed to the flower-like microspherical morphology, 30 high surface and narrow bandgap of the sample. The multiple reflections in microspheres leads to multi-reflection of the UV light, and trapping the photons in the samples. Compared to another two samples, S-P-2 possesses a larger contact area between the dyes and the synthetic crystal. This facilitates the 35 catalytic reactions on the semiconductor surface. Additionally, the electronic structures also benefits its photocatalytic reactivity. Compared with $\mathrm{SbPO}_{4}$ prepared at $140{ }^{\circ} \mathrm{C}$ or $180{ }^{\circ} \mathrm{C}$, the $160{ }^{\circ} \mathrm{C}$ fabricated microspheres has a narrower band gaps given by UVVis spectrum (Figure.6) but lower electron-hole recombination 40 rate shown in the PL spectrum (Figure.7).All these three factors prompts S-P-2 as the best photocatalyst among the peers.
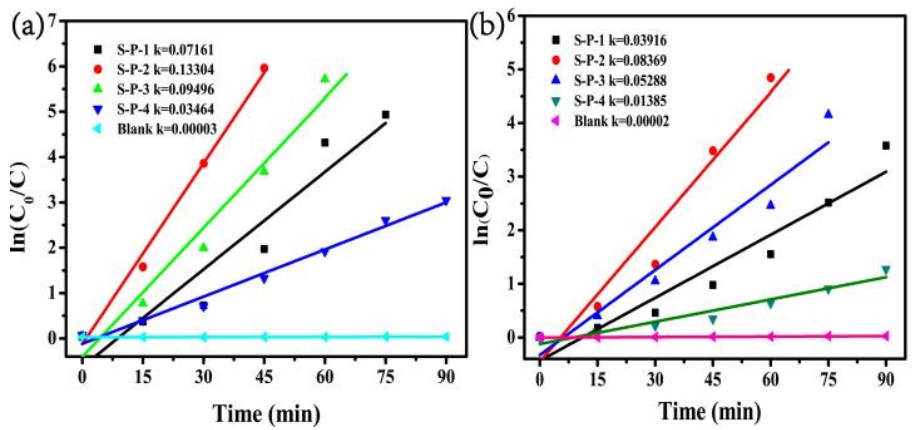

Figure 11. The pseudo-first-order kinetic model for the degradation of $\mathrm{RhB}$ (a) and $\mathrm{MB}$ (b) by $\mathrm{SbPO}_{4}$ photocatalysts.

${ }_{45} \mathrm{We}$ also tested the reusability of the $\mathrm{SbPO}_{4}$ samples. Figure 12 shows the photocatalytic ability of $\mathrm{RhB}$ over $\mathrm{SbPO}_{4}$ under UV light irradiation for five cycles. After 5 photocatalysis iterations, S-P-2 still kept significant photocatalytic robust with a degradation percentage of $94 \%$, confirming that the S-P-2 has 50 good photostability under UV light.

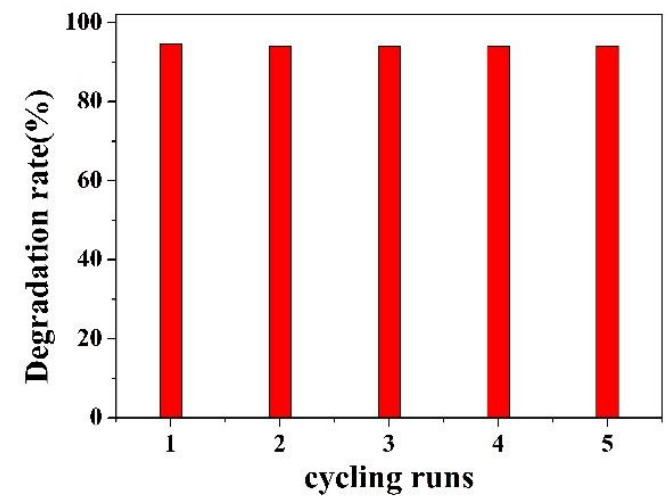

Figure 12. Cycling runs of S-P-2 for the photocatalytic degradation of $\mathrm{RhB}$.

To understand the photocatalytic of $\mathrm{SbPO}_{4}$, it is very important to 55 detect main reactive species (such as $\mathrm{h}+, \cdot \mathrm{OH}$ and $\mathrm{O}_{2}^{-}$radicals) that are involved in the photocatalytic process. In the radical and hole trapping experiments, EDTA-Na2, isopropanol (IPA) and pbenzoquinone (BQ) were used as scavengers for $h+, \cdot \mathrm{OH}$ and $\mathrm{O}_{2}{ }^{-}$respectively. As shown in Figure 13, when no quenchers were 60 added, the degradation efficiency of RhB was $99 \%$. When IPA and $\mathrm{AgNO}_{3}$ quencher were added, the $\mathrm{RhB}$ degradation efficiency was significantly reduced to $26 \%$ and $73 \%$. When EDTA-Na2 and p-benzoquinone (BQ) were added, the degradation efficiency of $\mathrm{RhB}$ decreased to $11 \%$ and $6 \%$, 65 respectively. These results indicate that $\mathrm{O}_{2}{ }^{-}$and $\mathrm{h}+$ are the main active oxidizing species, and the order of affecting degradation efficiencies of $\mathrm{RhB}$ follows that of $\mathrm{O}_{2}^{-}>\mathrm{h}+>\cdot \mathrm{OH}>\mathrm{e}^{-}$similar to the results from other groups.

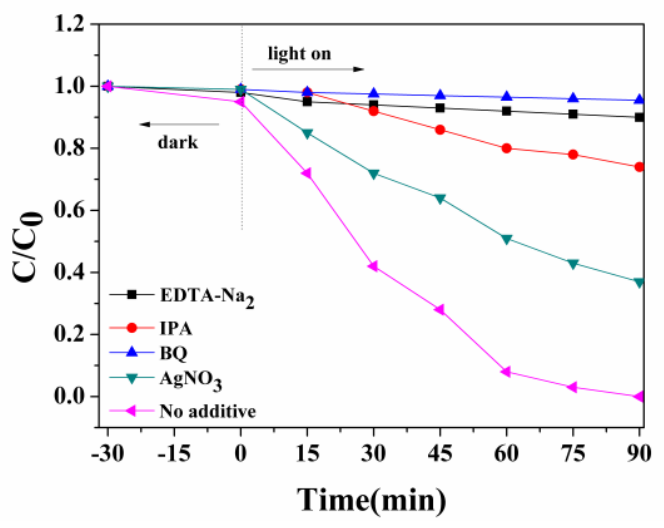

6| Journal Name, [year], [vol], 00-00

This journal is (C) The Royal Society of Chemistry [year] 
Figure 13. Effect of different quenchers on the RhB photodegradation by $\mathrm{SbPO}_{4}$ under visible-light irradiation for 90 min.

The photocatalytic mechanism is proposed as follows. Under UV 5 irradiation, the electrons originated from $\mathrm{SbPO}_{4}$ which react with $\mathrm{O}_{2}$ to form $\mathrm{O}_{2}{ }^{-}$radicals. $\mathrm{O}_{2}^{-}$can further convert to $\cdot \mathrm{OOH}$ and $\cdot \mathrm{OH}$. On the other hand, electrons could react with $\mathrm{O}_{2}$ and $\mathrm{H}^{+}$ to generate $\mathrm{H}_{2} \mathrm{O}_{2}$, which could also transform into $\cdot \mathrm{OH}$. Moreover, the generated $\mathrm{h}^{+}$of $\mathrm{SbPO}_{4}$ directly oxidizes $\mathrm{RhB}$ 10 molecules rather than form $\cdot \mathrm{OH}$ to indirectly oxidize $\mathrm{MB}$ molecules. The photocatalytic process can thus be schematized as follows:

$$
\begin{aligned}
& \mathrm{SbPO}_{4}+\mathrm{hv} \rightarrow \mathrm{SbPO}_{4}\left(\mathrm{e}^{-}+\mathrm{h}+\right) \\
& \mathrm{SbPO}_{4}\left(\mathrm{e}^{-}\right)+\mathrm{O}_{2} \rightarrow \mathrm{SbPO}_{4}+\cdot \mathrm{O}_{2}^{-} \\
& 15 \cdot \mathrm{O}_{2}{ }^{-}+\mathrm{H}_{2} \mathrm{O} \rightarrow \cdot \mathrm{OOH}+\mathrm{OH}^{-} \\
& \mathrm{e}^{-}+\mathrm{O}_{2}+2 \mathrm{H}^{+} \rightarrow \mathrm{H}_{2} \mathrm{O}_{2} \\
& \mathrm{H}_{2} \mathrm{O}_{2}+\cdot \mathrm{O}_{2} \rightarrow \cdot \mathrm{OH}+\mathrm{OH}^{-}+\mathrm{O}_{2} \\
& \mathrm{MB} / \mathrm{RhB}+\mathrm{h}++\cdot \mathrm{OH}+\cdot \mathrm{O}_{2} \rightarrow \text { degradation products }
\end{aligned}
$$

\section{Conclusions}

20 In summary, we have devised an hydrothermal synthesis to fabricate flower and sphere-likeSbPO 4 with $[\mathrm{BMIM}]\left[\mathrm{PO}_{4}\right]$ as a coupling agent. The spherical shapes can be simply controlled through changing the hydrothermal reaction temperature. UV-Vis results shows that the bandgap of for S-P-1, S-P-2, S-P-3, S-P-4

25 was about $3.19,2.80,4.12$ and $4.71 \mathrm{eV}$, respectively. The BET surface areas of the products have impact on the degradation of the as-synthesized samples. When used for the photocatalytic degradation of $\mathrm{RhB}$ and $\mathrm{MB}$ under UV light irradiation, the $\mathrm{SbPO}_{4}$ prepared via ionic-liquid-assisted route demonstrate better 30 photocatalytic effects than the no-ionic-liquid-assisted counterparts. From characterizations and scavenger tests, the high photocatalytic ability of $\mathrm{SbPO}_{4}$ microspheres is attributed to the homogeneous spherical, relatively larger surface area and narrow band gap of the samples. The photocatalytic efficiency of RhB 35 and MB comply with the following order S-P-2>S-P-3>S-P-1>S$\mathrm{P}-4$. Moreover, S-P-2 shows the highest degradation performance of both $\mathrm{RhB}$ and $\mathrm{MB}$, considerably extending potential for research and practical applications.

\section{Acknowledgments}

40 The authors acknowledge with thanks the financial support of the National Natural Science Foundation of China (21601149) and Scientific Research Fund of Hunan Provincial Education Department, China (16B253), the Open Project Program of State Key Laboratory of Structural Chemistry, China (No. 20150018) 45 and Hunan 2011 Collaborative Innovation Center of Chemical Engineering \& Technology with Environmental Benignity and Effective Resource Utilization and the Oulu University Strategic Grant. T. Li acknowledges Oulu University Short-term International Research Visit grant during his stay in Finland.
${ }^{a}$ College of Chemistry, Key Lab of Environment Friendly Chemistry and Application in Ministry of Education, Xiangtan University, Xiangtan, 411105, China.Fax: 86-731-8292251; Tel: 86-731-58292206; E-mail: fengli_xtu@hotmail.com; hnlth@xtu.edu.cn.

${ }_{55}{ }^{b}$ Nano and Molecular Systems Research Unit, Faculty of Science, P. O. Box 3000, University of Oulu, FIN-90014, Finland

(1) R. G. Chen, J. H. Bi, L. Wu, Z. H. Li,; X. Z. Fu,., Crystal Growth \& Design, 2009,9, 1775-1779.

60 (2) S. Y. Wu, H. Zheng, Y. Y. Wu, W. Lin, T. Z. Xu, Guan, M., Ceram Int, 2014, 40, 14613-14620.

(3) X. Chen, D. X. Zhao, K. W. Liu, C. R. Wang, B.H. Li, Z.Z. Zhang, D. Z. Shen, , ACS Appl Mater Inter, 2015,7, 16070-16077.

(4) J. H. Sun, H. Yang, Ceram Int, 2014, 40, 6399-6404.

${ }_{65}$ (5) X. Zhao, W. Q. Yao, Y. Wu, S. C. Zhang, H. P. Yang, Y. F. Zhu, , J Solid State Chem, 2006,179, 2562-2570.

(6) G. Q. Zhu, W. X Que, J Clust Sci, 2013, 24, 531-547.

(7) X. F. Zhang, L. L. Du, H. Wang, X. L. Dong, X. X. Zhang, C. Ma, H. C. Ma, MicroporMesopor Mat, 2013,173, 175-180.

70 (8) I. Paramasivam, J. M. Macak, T. Selvam, P. Schmuki, ElectrochimActa, 2008, 54, 643-648.

(9) X. Z. Liu, W. Si, C. Ding, S. L. Zang, Chinese J InorgChem, 2004, $20,1445-1448$

(10) G. Alonzo, N. Bertazzi, P. Galli, G. Marci, M. A. Massucci, L. 75 Palmisano, P. Patrono, F. Saiano, Mater Res Bull, 1998,33, 1233-1240.

(11) S. Y. Wu, H. Zheng, Y. W. Lian,; Y. Y. Wu, Mater Res Bull, 2013,48, 2901-2907.

(12) X. R. Yan, T. Bai, M. L. Huo, Y. P. Zhang, X. Y. Guo, Chinese J Catal, 2004,25, 120-124.

80 (13) Y. Huang, G. Xie, S. Chen, S. Gao, J Solid State Chem, 2011, 184, 502-508.

(14) X. Lin, F. Huang, W. Wang, Y. Wang, Y. Xia, J. Shi, ApplCatal A: Gen, 2006,313, 218-223.

(15) M. Ahlawat, G. V. Vazquez, M. Nalin, Y. Messaddeq, S. Ribeiro, R. ${ }_{85}$ Kashyap, J Non-Cryst Solids, 2010, 356, 2360-2362.

(16) Q. Han, L. Chen, M. Wang, X. Yang, L. Lu, X. Wang, Mat SciEng $B$, 2010, 166, 118-121.

(17) S. P. Hu, C.Y Xu. F. X. Ma, L. Cao, L. Zhen, Dalton T, 2014, 43, 8439-8445.

90 (18) D.N Liu, G. H. He, L. Zhu, W.Y. Zhou, Y.H. Xu, Appl Surf Sci, 2012,258, 8055-8060.

(19) Y. Wang, L. li, G. Li, RSC Adv, 2012, 2, 12999-13006.

(20) J. B. Biswal, S. S. Garje, N. Revaprasadu, Polyhedron, 2014, 80, 216-222.

95 (21) L. Qi, CoordinChem Rev, 2010,254, 1054-1071.

(22) K. S. Yao, W. W. Lu, J. J. Wang, Mater ChemPhys, 2011, 130, $1175-1181$

(23) Y. Z. Wu, X. P. Hao, J. X. Yang, F. Tian, M. H. Jiang, , Mater Lett, 2006,60, 2764-2766.

100 (24) S. L. Yu, B. C. Liu, Q. Wang, Y. X. Gao, Y. Shi, X. Feng, X. T. An, L. X. Liu, J. Zhang, ACS Appl Mater Inter, 2014,6, 10283-10295.

(25) T. Sivakumar Natarajan, H. C. Bajaj, R. J. Tayade, , Crystengcomm ,2015, 17, 1037-1049.

(26) H. Zhang, Q. Zhu, Y. Zhang, Y. Wang, L. Zhao, B. Yu, Adv Funct 105 Mater, 2007,17, 2766-2771.

(27) V. Sudarsan, K. P. Muthe, J. C. Vyas, S. K. Kulshreshtha, J Alloy Compd, 2002,336, 119-123.

(28) Y. N. Guo, X. Yang, F. Y. Ma, K. X. Li, L. Xu, X. Yuan, Y. H. Guo, Appl Surf Sci, 2010,256, 2215-2222.

110 (29) B. Pan, Y. Wang, Y. Y. Liang, S. J. Luo, W. Y. Su, X. X. Wang, Int J Hydrogen Energ, 2014,39, 13527-13533.

(30) C. S. Pan, Y. F. Zhu, J Mater Chem, 2011, 21, 4235-4241.

(31) S. Vyas, C .Dreyer, J. Slingsby, et al. Journal of Physical Chemistry A, 2014, 118(34):6873.

115 (32) G. H. Liu, S. W .Liu, Q. F. Lu, H. Y. Sun, Z. L. Xiu, IndEngChem Res, 2014,53, 13023-13029.

(33) B.S. Naidu, V. Sudarsan, R. K. Vatsa, Journal of Nanoscience \& Nanotechnology, 2009, 9(5):2997.

(34) C. Yang, L. Zhang, Z. Wang, et al. Materials Science \& Engineering 120 B, 2016, 207:39-46.

(35) M. Kruk, M. Jaroniec, Chem Mate, r2001, 13, 3169-3183.

\section{${ }_{50}$ Notes and references}

